\title{
Successful Multidisciplinary Treatment with Secondary Metastatic Liver Resection after Downsizing by Palliative Second-Line Treatment of Colorectal Cancer: A Curative Option
}

\author{
Axel Wein $^{\mathrm{a}} \quad$ Jürgen Siebler ${ }^{\mathrm{a}} \quad$ Ruediger Goertz $^{\mathrm{a}} \quad$ Kerstin Wolff $^{\mathrm{a}}$ \\ Nicola Ostermeier ${ }^{a} \quad$ Dagmar Busse $^{a}$ Andreas E. Kremer ${ }^{a} \quad$ Franz Koch $^{a}$ \\ Alexander Hagel $^{a} \quad$ Michael Farnbacher $^{d}$ Ferdinand J. Kammerer $^{b}$ \\ Markus F. Neurath ${ }^{a}$ Robert Gruetzmann ${ }^{c}$

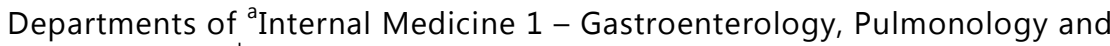 \\ Endocrinology, ${ }^{b}$ Radiology, and ${ }^{c}$ Surgery, Friedrich-Alexander University \\ Erlangen-Nuremberg, and ${ }^{\mathrm{d}}$ Practice of Gastroenterology, Erlangen, Germany
}

\section{Key Words}

Colorectal cancer · Secondary liver metastasis resection · Second-line chemotherapy · Curative option

\begin{abstract}
Introduction: The prognostic outcome following progression after palliative first-line treatment for patients suffering from metastatic colorectal adenocarcinoma is generally poor. Long-term relapse-free survival with palliative second-line treatment may be achieved in only a limited number of individual cases. Case Report: A 37-year-old patient presented with bilobar liver metastases of colon cancer confirmed by histology with wild-type K-RAS (exon 2). Due to progressive disease after eight cycles of first-line therapy with FOLFIRI plus cetuximab, second-line chemotherapy with modified FOLFOX4 (mFOLFOX4) plus bevacizumab was initiated. During four cycles of mFOLFOX4 plus bevacizumab (2 months), no higher-grade toxicity occurred. Liver MRI with contrast medium revealed downsizing of the segment II/III metastases, as well as regressive, small, faint, hardly definable lesions in segments VI and IVb. The interdisciplinary tumor board of the University of Erlangen thus decided to perform re-
\end{abstract}

\section{KARGER}

Prof Dr med Axel Wein

Department of Internal Medicine 1 - Gastroenterology, Pulmonology and

Endocrinology, Friedrich-Alexander University Erlangen-Nuremberg

Ulmenweg 18, DE-91054 Erlangen (Germany)

E-Mail axel.wein@uk-erlangen.de 
Wein et al.: Successful Multidisciplinary Treatment with Secondary Metastatic Liver Resection after Downsizing by Palliative Second-Line Treatment of Colorectal Cancer

section of the liver metastases. Segments II and III were resected, and the liver metastases in segments IVa and VI were excised (R0). Histopathology confirmed three of the R0-resected metastases to be completely necrotic, with residual scarring. As perioperative therapy, four additional cycles of mFOLFOX4 plus bevacizumab were administered postoperatively. No higher-grade toxicity was observed. Three years after the initial diagnosis, the patient is relapse free, professionally fully reintegrated, and has an excellent performance status. Conclusion: Patients suffering from metastatic colorectal cancer may benefit from multidisciplinary treatment with secondary metastatic liver resection after downsizing by palliative second-line treatment. In individual cases, patients may even have a curative treatment option, provided that close interdisciplinary collaboration exists.

(C) 2016 The Author(s)

Published by S. Karger AG, Basel

\section{Introduction}

Colorectal cancer (CRC) is the second most common form of cancer in women and the third most common cancer in men. In 2008, the estimated worldwide incidence of CRC exceeded 1.2 million, with a mortality rate of over 600,000 [1]. Also in Germany, CRC is one of the most common malignancies; more than 70,000 new cases and approximately 30,000 mortalities are recorded each year [2]. Metastases at diagnosis are seen in about 20-25\% of all CRCs, and the majority of cases are not amenable to primary curative resection [2]. Recent studies have demonstrated substantial improvements in overall survival (OS) achieved by the inclusion of new agents, 'biologicals', in palliative first-line treatment approaches [35], as well as by close interdisciplinary cooperation with the objective of secondary metastasis resection [6-8].

The multimodal approach of secondary metastasis resection following downsizing by first-line treatment achieves enormous improvements in median survival times, which individual studies have reported at 49,53.9, and 60 months, respectively, in patients receiving multimodality treatment [6-8]. A multimodal approach in the sense of secondary metastasis resection after downsizing by palliative second-line therapy has not been described until now [7], despite the fact that new situations have recently also arisen in second-line treatments owing to the inclusion of new agents [9-12].

\section{Case Report}

A 37-year-old patient in very good general condition (ECOG grade 0 , weight $87 \mathrm{~kg}$, height $175 \mathrm{~cm}$ ) presented with mid-abdominal symptoms which had been experienced for 2 months. Upon further imaging evaluation, sonography and contrast-enhanced abdominal CT revealed several hypodense lesions with a bilobar distribution, initially $2 \times 2 \mathrm{~cm}$ in size, which were considered to be suspected metastases. Subsequent colonoscopy identified an ulcerous lesion in the cecum, and a biopsy sample was taken. Histopathologic analysis confirmed a moderately differentiated adenocarcinoma. Esophagogastroduodenoscopy and laboratory tests including the tumor markers carcinoembryonic antigen (CEA) und cancer antigen (CA) 19-9 revealed no pathological findings. The case was subsequently presented to the interdisciplinary tumor board, and palliative colon carcinoma resection with intraoperative biopsy sampling of the liver lesion for confirmation of suspected liver metastases was recommended. 
Wein et al.: Successful Multidisciplinary Treatment with Secondary Metastatic Liver Resection after Downsizing by Palliative Second-Line Treatment of Colorectal Cancer

Palliative right-side hemicolectomy was performed on October 23, 2012, and multiple punch biopsies of the largest liver lesion in segment II were taken intraoperatively. Histopathology confirmed category: pT3, pN0 (0 /64), L0, V0, Pn0, cMx, locally resected with negative margins, overall an RX situation. Histopathologic analysis of biopsy samples taken from the segment II liver lesion revealed only necrotic portions with no evidence of malignancy, as well as inflammatory fibrotic changes and a predominantly microvascular steatosis of the liver $(<20 \%$ ). Wild-type K-RAS (exon 2) was additionally analyzed in the primary tumor. The patient's postoperative recovery was as expected, and the case was again discussed by the interdisciplinary tumor board. Repeated sonographically guided biopsy of a liver lesion was recommended. Additional gadolinium-enhanced MRI also revealed multiple suspected malignant liver lesions in segments II, IVb, and VI, as well as hepatic steatosis. There was no evidence of liver cirrhosis and $\alpha$-fetoprotein was normal.

Ultrasound-guided punch biopsies of the liver lesion in segment II were taken on February 13,2013 . Histopathologic evaluation of the two biopsy samples, including their stepwise workup, again revealed no atypical cells.

During closely monitored follow-up, MRI with Primovist ${ }^{\circledR}$ on April 9, 2013, revealed bilobar enlargement of the liver lesions, e.g. in segment II from $1.7 \times 2.1$ to $1.8 \times 2.5 \mathrm{~cm}$. Following renewed discussion of the case by the tumor board, the strong clinical suspicion of liver metastases from K-RAS wild-type colon carcinoma led to the recommendation to initiate palliative irinotecan-based first-line combination chemotherapy with FOLFIRI concomitant with anti-EGFR therapy.

After fully informing the patient about the procedure and implantation of a portacath, palliative first-line treatment with the FOLFIRI regimen plus cetuximab was initiated. From April 9, 2013, on day 1 of a 14-day treatment cycle, the patient received cetuximab (initial dose $400 \mathrm{mg} / \mathrm{m}^{2}$ of body surface area infused over $120 \mathrm{~min}$, thereafter $250 \mathrm{mg} / \mathrm{m}^{2}$ of body surface area infused over 60 min weekly) plus FOLFIRI (comprising a 60- to 90-min infusion of irinotecan at a dose of $180 \mathrm{mg} / \mathrm{m}^{2}$ of body surface area, a 120 -min infusion of racemic folinic acid at a dose of $400 \mathrm{mg} / \mathrm{m}^{2}$ of body surface area, and 5 -fluorouracil as an i.v. bolus of $400 \mathrm{mg} / \mathrm{m}^{2}$ of body surface area, and then a continuous 46 -hour infusion of $2,400 \mathrm{mg} / \mathrm{m}^{2}$ of body surface area). Supportive therapy comprised prior administration of Zofran ${ }^{\circledR}(8 \mathrm{mg}$ i.v.) and Fortecortin ${ }^{\circledR}$ (8 mg i.v.) as well as Tavegil ${ }^{\circledR}(2 \mathrm{mg}$ i.v. $)$ and Zantic ${ }^{\circledR}(5 \mathrm{ml}$ i.v.) before cetuximab application. Atropine was applied subcutaneously $(0.25 \mathrm{mg})$. After four fortnightly cycles of FOLFIRI plus cetuximab (2 months), follow-up MRI with Primovist ${ }^{\circledR}$ showed a small enlargement of the indicator lesion in segment II to $1.9 \times 2.6 \mathrm{~cm}$. The overall situation was stable disease. Apart from grade I CTC (Common Toxicity Criteria) toxicity (diarrhea), treatment was well tolerated, and four more cycles of FOLFIRI plus cetuximab (2 months) were administered. Subsequent follow-up with liver MRI on August 26, 2013, revealed enlargement of the indicator lesion to $2.6 \times 2.8 \mathrm{~cm}$ (fig. 1). The CEA and CA 19-9 tumor markers remained unchanged and slightly increased. The main toxicity was grade I acneiform dermatitis. After presentation of the case to the interdisciplinary tumor board, the recommendation was made, in the presence of progressive disease, to change to palliative second-line treatment with the modified FOLFOX4 (mFOLFOX4) regimen plus biweekly bevacizumab. From August 26, 2013, a total of four cycles (2 months) of mFOLFOX4 plus bevacizumab were completed according to the treatment plan, and no higher-grade toxicity was observed. On day 1 of a 14-day treatment cycle, the patient received bevacizumab $5 \mathrm{mg} / \mathrm{kg}$, oxaliplatin $85 \mathrm{mg} / \mathrm{m}^{2}$ of body surface area as a 2-hour infusion, a 120 -min infusion of $200 \mathrm{mg} / \mathrm{m}^{2}$ racemic folinic acid, and then 5 -fluorouracil $3,200 \mathrm{mg} / \mathrm{m}^{2}$ of body surface area as a continuous 48-hour infusion via a pump system. Follow-up liver MRI (fig. 2) on November 4, 2013, showed that the reference lesions in segments II/III were significantly reduced to current 
Wein et al.: Successful Multidisciplinary Treatment with Secondary Metastatic Liver Resection after Downsizing by Palliative Second-Line Treatment of Colorectal Cancer

RECIST score 2.9, whereas the score was 4.3 in the pretreatment MRI, representing a partial response. Thoracic CT with i.v. contrast medium on November 4, 2013, revealed no suspected lung metastases; the CEA und CA 19-9 tumor markers were still slightly raised. Following presentation of the images to the interdisciplinary tumor board, surgical resection of the liver metastases was recommended. On November 29, 2013, following laparotomy and intraoperative liver sonography, segments II and III were resected, and local excision of segments IVa and VI was performed. The synopsis of the histopathologic workout of the metastases included a small subcapsular locus with sparse residual vital infiltration of a moderately differentiated adenocarcinoma, which corresponds well to subnecrotic liver metastasis of cecal carcinoma, as well as three further completely necrotic loci which were all resected with negative margins (R0). Postsurgical healing was without complications. The patient subsequently received four additional cycles of mFOLFOX4 in a perioperative setting, and no higher-grade toxicity was observed.

Thereafter, cancer follow-up comprising liver sonography and tumor marker analysis was performed at 3-month intervals. The patient had a good performance status and was professionally fully reintegrated. The most recent liver MRI (fig. 3) and thoracic CT performed on May 13, 2015, revealed no indications of disease activity. Ongoing follow-up is now being performed by a practicing gastroenterologist, and there was no evidence of disease at the last appointment on November 2, 2015.

\section{Discussion}

A meta-analysis of first-line therapy in CRC was able to demonstrate a significant correlation of response rate and metastasis localization (liver-only metastasis) with the rate of secondary metastasis resection [14]. Additional patient characteristics associated with an increased probability of secondary metastasis resection have not yet been validated for firstline treatment [14]. It can be expected that the factors 'response rate' and 'liver-only situation' also play a role in the case of secondary metastasis resection after downsizing by second-line treatment. Investigations into secondary metastasis resection from previous large phase III studies on second-line therapy are currently lacking [9-13]. According to current studies, the most favorable starting point for secondary metastasis resection, i.e. a liver-only situation before second-line treatment, is relatively common. In the VELOUR study, 25\% of patients had a liver-only situation before initiations of second-line treatment [10]; in the RAISE study, this figure was 32\% [11]. The proportion of patients with liver-only metastases at the beginning of second-line treatment was also 32\% in the FIRE3 study [15].

Curative secondary metastasis resection following second-line treatment has not been described until now. In this case, the treatment decision was taken in favor of mFOLOFOX4 plus bevacizumab as a second-line therapy following progression after FOLFIRI plus cetuximab as a first-line treatment. This decision was based on the fact that in the large E3200 phase III study, this combination achieved a high response rate of $20 \%$ among patients pretreated with irinotecan-based combination chemotherapy, and other primary and secondary outcome criteria (OS and progression-free survival) were also significantly improved [9]. In the FIRE3 study, which also investigated patients with wild-type K-RAS (exon 2), first-line treatment with FOLFIRI plus cetuximab was often followed by second-line treatment with FOLFOX4 plus bevacizumab. This study achieved a response rate of $19.7 \%$ and a median OS of 16.3 months [15], which demonstrates the efficiency of this combination as a second-line treatment following progression after FOLFIRI plus cetuximab in wild-type K-RAS tumors. Secondary metastasis resection was not performed following second-line treatment [15]. 
Wein et al.: Successful Multidisciplinary Treatment with Secondary Metastatic Liver Resection after Downsizing by Palliative Second-Line Treatment of Colorectal Cancer

Recently, the phase III TML study on second-line treatment in CRC demonstrated that for first-line treatment with combination chemotherapy plus bevacizumab, the response rate to second-line treatment could only be increased slightly by the combination of chemotherapy plus bevacizumab, from 4 to 5.4\% [12]. Following progression after first-line treatment, the prognosis of patients with metastasized CRC is limited, despite new substances licensed for use in second-line treatments [10-12]. In the era of combination chemotherapy, median survival was, for example, 10 months with FOLFIRI after prior treatment with FOLFOX6 [15]; in the TML study with bevacizumab, OS was only slightly better, at 11.3 months [12]. By using a combination of FOLFIRI plus aflibercept after pretreatment with oxaliplatinbased combination chemotherapy, a median survival of 13 months was achieved [13]. This was also true of the FOLFIRI plus ramucirumab combination following pretreatment with FOLFOX4 plus bevacizumab in the RAISE study [11]. In patients with wild-type K-RAS tumors, the data on second-line treatment are limited. In the FIRE3 study, a median survival of 16.3 months could be reached with the combination of FOLFOX4 plus bevacizumab [15].

The likelihood of potential cure following progression after first-line treatment is very low, since imaging during second-line treatment reveals that the rate of complete remission is as low as $0-4 \%$ [10-13]. It may be possible to increase the proportion of complete remissions by intensifying interdisciplinary collaborations to enable curative secondary metastasis resection following downsizing (partial response after second-line treatment plus curative R0 secondary metastasis resection). In the current patient, survival (OS) from the start of second-line treatment was 27 months, and the time with no evidence of disease after curative secondary metastasis resection was 24 months. The OS achieved by the current patient is thus significantly improved compared to the situation with systemic treatment alone [9-11,13]. Furthermore, the approach offers the patient a curative option.

To the best of the authors' knowledge, the literature contains no previous reports of secondary metastasis resection after downsizing with palliative second-line therapy in CRC, and also no systematic prospective studies. One retrospective study reports on 60 patients whose data had been collected over a period of 11 years [16]. This previously published investigation on secondary metastasis resection following second-line treatment by Brouquet et al. [16] includes 60 patients with metastasized CRC. The median follow-up was 32 months. No data on response to second-line treatment or on median survival of the entire collective were reported. The regimens used in second-line treatment were highly inhomogeneous [16]. The 1- and 5-year survival rates were 83 and 22\%, respectively. Of the 60 patients, $8(13 \%)$ were free of disease after 12 months. The latter study thus demonstrates that secondary metastasis resection following second-line therapy seems to be associated with a prognostic benefit [16].

\section{Conclusion}

Patients suffering from metastatic CRC may benefit from a multidisciplinary approach after downsizing of liver metastases by second-line chemotherapy. This multimodal approach can be successfully applied following a response to palliative second-line treatment, and a curative option for these patients may be possible. Regular presentation of imaging findings to an interdisciplinary tumor board highly experienced in surgical oncology is important for enabling the prognosis of these patients to be improved in a multimodal approach. 
Wein et al:: Successful Multidisciplinary Treatment with Secondary Metastatic Liver Resection after Downsizing by Palliative Second-Line Treatment of Colorectal Cancer

\section{Statement of Ethics}

The case report met the standards laid down in the Helsinki Declaration of 1975 (in its current, revised form). Written informed consent was obtained from the patient.

\section{Disclosure Statement}

The authors declare that they have no conflicts of interest. All authors have disclosed any possible conflicts of interest.

\section{References}

1 Ferlay J, Shin HR, Bray F, Forman D, Mathers C, Parkin DM: Estimates of worldwide burden of cancer in 2008: GLOBOCAN 2008. Int J Cancer 2010;15:2893-2917.

-2 Pox C, Aretz S, Bischoff C, Graeven U, Hass M, Heussner P, Hohenberger W, Holstege A, Hübner J, Kolligs F, Kreis M, Lux P, Ockenga J, Porschen R, Post S, Rahner N, Reinacher-Schick A, Riemann JF, Sauer R, Sieg A, Scheppach W, Schmitt W, Schmoll HJ, Schulmann K, Tannapfel A, Schmiegel W: S3-Leitlinie kolorektales Karzinom Version 1.0 - Juni 2013. S3-Guideline Colorectal Cancer Version 1.0. Z Gastroenterol 2013;51:753-854.

-3 Van Cutsem E, Köhne CH, Láng I, Folprecht G, Nowacki MP, Cascinu S, Shchepotin I, Maurel J, Cunningham D, Tejpar S, Schlichting M, Zubel A, Celik I, Rougier P, Ciardello F: Cetuximab plus irinotecan, fluorouracil, and leucovorin as first-line treatment for metastatic colorectal cancer: updated analysis of overall survival according to tumor KRAS and BRAF mutation status. J Clin Oncol 2011;29:2011-2019.

-4 Heinemann V, von Weikersthal LF, Decker T, Kiani A, Vehling-Kaiser U, Al-Batran SE, Heintges T, Lerchenmueller C, Kahl C, Seipelt G, Kullmann F, Stauch M, Scheithauer W, Hielscher J, Scholz M, Mueller S, Link H, Niederle N, Rost A, Höffkes HG, Moehler M, Lindig RU, Modest DP, Rossius L, Kirchner T, Jung A, Stintzing S: FOLFIRI plus cetuximab versus FOLFIRI plus bevacizumab as first-line treatment for patients with metastatic colorectal cancer (FIRE3): a randomised, open-label, phase 3 trial. Lancet Oncol 2014;15:1065-1075.

5 Stintzing S, Jung A, Rossius L, Modest DP, Fischer bon Weikersthal L, Decker T, Möhler M, Scheithauer W, Kirchner T, Heinemann V: Analysis of KRAS/NRAS and BRAF mutations in FIRE-3: A randomized phase III study of FOLFIRI plus cetuximab or bevacizumab as first-line treatment for wild-type (WT) KRAS (exon 2) metastatic colorectal cancer (mCRC) patients. J Cancer 2013;49(suppl 3):s8-s9.

-6 Venook A, Niedzwiecki D, Lenz H, Mahoney M, Innocenti F, O’Neil B, Hochster H, Goldberg R, Schilsky R, Mayer R, Polite B, Atkins J, Shaw JE, Bertagnolli M, Blanke C: LBA10 CALGB/SWOG 80405: Analysis of patients undergoing surgery as part of treatment strategy. Ann Oncol 2014;25:v1-v41.

-7 Wein A, Emmert M, Merkel S, Harich HD, Siebler J, Thiemann R, Lamberti C, Göttler B, Fries S, Kiani A, Schlag R, Grüner M, Steinbild S, Eberl S, Pohl-Dernick K, Dörje F, Horbach T, Schöffski O, Neurath MF, Hohenberger W: Palliative treatment of colorectal cancer with secondary metastasis resection in Germany - impact of the multidisciplinary treatment approach on prognosis and cost: the Northern Bavaria IVOPAK I Project. Oncology 2014;88:103-121.

-8 Folprecht G, Gruenberger T, Bechstein W, Raab HR, Weitz J, Lordick F, Hartmann JT, Stoehlmacher-Williams J, Lang H, Trarbach T, Liersch T, Ockert D, Jaeger D, Steger U, Suedhoff T, Rentsch A, Köhne CH: Survival of patients with initially unresectable colorectal liver metastases treated with FOLFOX/cetuximab or FOLFIRI/cetuximab in a multidisciplinary concept (CELIM study). Ann Oncol 2014;25:1018-1025.

-9 Giantonio BJ, Catalano PJ, Meropol NJ, O’Dwyer PJ, Mitchell EP, Alberts SR, Schwartz MA, Benson Ill AB: Bevacizumab in combination with oxaliplatin, fluorouracil, and leucovorin (FOLFOX4) for previously treated metastatic colorectal cancer: results from the Eastern Cooperative Oncology Group Study E3200. J Clin Oncol 2007;25:1539-1544.

10 Van Cutsem E, Tabernero J, Lakomy R, Prenen H, Prausová J, Macarulla T, Ruff P, van Hazel GA, Moiseyenko V, Ferry D, McKendrick J, Polikoff J, Tellier A, Castan R, Allegra C: Addition of aflibercept to fluorouracil, leucovorin, and irinotecan improves survival in a phase III randomized trial in patients with metastatic colorectal cancer previously treated with an oxaliplatin-based regimen. J Clin Oncol 2012;30:3499-3506.

-11 Tabernero J, Yoshino T, Cohn AL, Obermannova R, Bodoky G, Garcia-Carbonero R, Ciuleanu TE, Portnoy D, Van Cutsem E, Grothey A, Prausová J, Garcia-Alfonso P, Yamazaki K, Clingan PR, Lonardi S, Kim TW, Simms L, Chang SC, Nasroulah F; RAISE Study Investigators: Ramucirumab versus placebo in combination with second-line FOLFIRI in patients with metastatic colorectal carcinoma that progressed during or after first- 
Wein et al:: Successful Multidisciplinary Treatment with Secondary Metastatic Liver Resection after Downsizing by Palliative Second-Line Treatment of Colorectal Cancer

line therapy with bevacizumab, oxaliplatin, and a fluoropyrimidine (RAISE): a randomised, double-blind, multicentre, phase 3 study. Lancet Oncol 2015;16:499-508.

12 Bennouna J, Sastre J, Arnold D, Osterlund P, Greil R, Van Cutsem E, von Moos R, Viéitez JM, Bouché O, Borg C, Steffens CC, Alonso-Orduña V, Schlichting C, Reyes-Rivera I, Bendahmane B, André T, Kubicka S; ML18147 Study Investigators: Continuation of bevacizumab after first progression in metastatic colorectal cancer (ML18147): a randomised phase 3 trial. Lancet Oncol 2013;14:29-37.

13 Tournigand C, André T, Achille E, Lledo G, Flesh M, Mery-Mignard D, Quinaux E, Couteau C, Buyse M, Ganem G, Landi B, Colin P, Louvet C, de Gramont A: FOLFIRI followed by FOLFOX6 or the reverse sequence in advanced colorectal cancer: a randomized GERCOR study. J Clin Oncol 2004;22:229-237.

-14 Folprecht G, Grothey A, Alberts S, Raab HR, Köhne CH: Neoadjuvant treatment of unresectable colorectal liver metastases: correlation between tumour response and resection rates. Ann Oncol 2005;16:1311-1319.

15 Modest DP, Stintzing S, Fischer von Weikersthal L, Decker T, Kiani A, Vehling-Kaiser U, Al-Batran SE, Heintges T, Lerchenmüller C, Kahl C, Seipelt G, Kullmann F, Stauch M, Scheithauer W, Held S, Möhler M, Jung A, Kirchner T, Heinemann V: Impact of subsequent therapies on outcome of the FIRE-3/AIO KRK0306 trial: first-line therapy with FOLFIRI plus cetuximab or bevacizumab in patients with KRAS wild-type tumors in metastatic colorectal cancer. J Clin Oncol 2015;33:3718-3726.

16 Brouquet A, Overman MJ, Kopetz S, Maru DM, Loyer EM, Andreou A, Cooper A, Curley SA, Garrett CR, Abdalla EK, Vauthey J-N: Is resection of colorectal liver metastases after a second-line chemotherapy regimen justified? Cancer 2011;117:4484-4492.

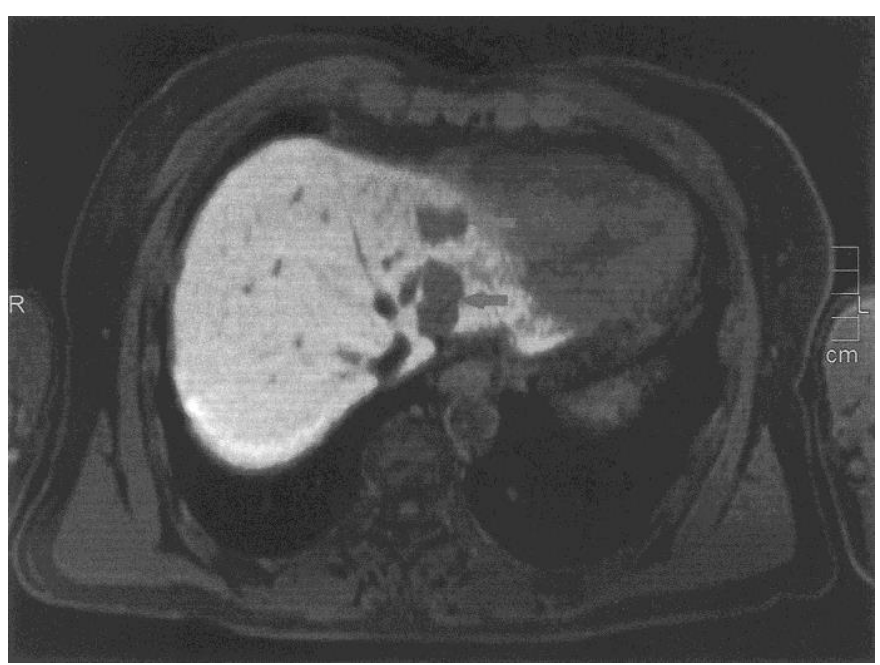

Fig. 1. Liver MRI with contrast medium (August 26, 2013). T1-weighted MRI in an axial plane; liver metastases in segments II and III before initiating second-line chemotherapy with mFOLFOX4 plus bevacizumab. 


\section{Case Reports in Oncology}

\begin{tabular}{l|l}
\hline \multicolumn{2}{l}{ Case Rep Oncol 2016;9:379-386 } \\
\hline DOI: 10.1159/000445677 & $\begin{array}{l}\text { (c) 2016 The Author(s). Published by S. Karger AG, Basel } \\
\text { www.karger.com/cro }\end{array}$ \\
\hline
\end{tabular}

Wein et al.: Successful Multidisciplinary Treatment with Secondary Metastatic Liver Resection after Downsizing by Palliative Second-Line Treatment of Colorectal Cancer

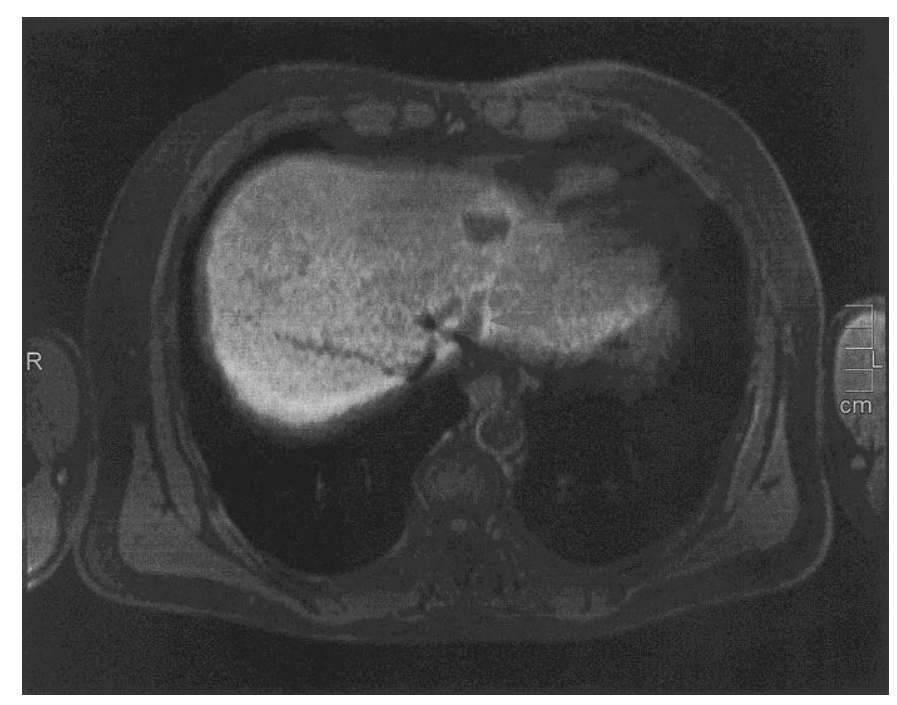

Fig. 2. Liver MRI with contrast medium (November 4, 2013). T1-weighted MRI in an axial plane; partial remission of liver metastases in segment III (arrows) after four cycles of second-line chemotherapy with mFOLFOX4 plus bevacizumab.

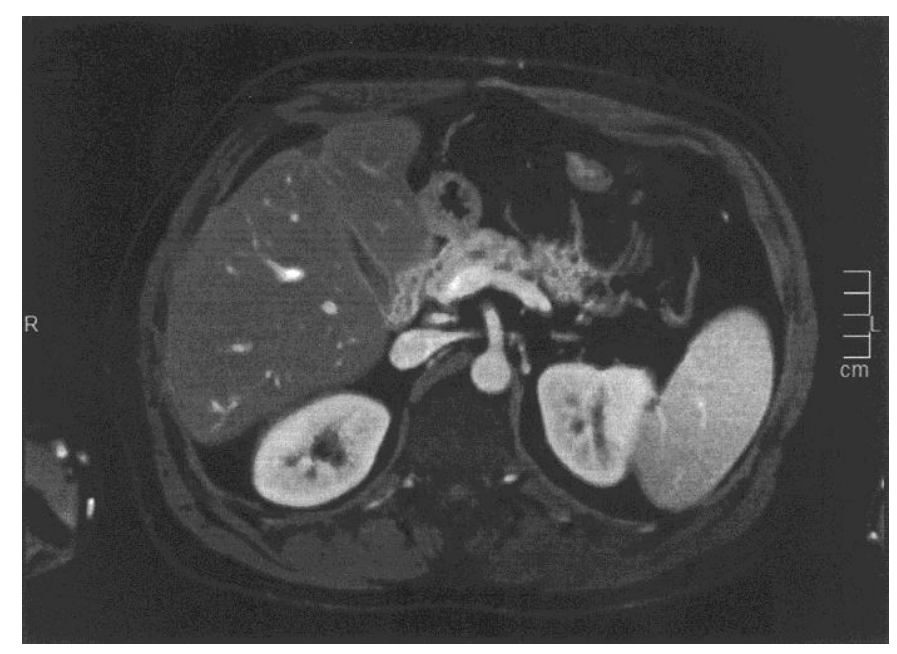

Fig. 3. Liver MRI with contrast medium (May 19, 2014). T1-weighted MRI in an axial plane; follow-up after hepatic resection of segments II and II and local excision of metastases in segments IVb and VI. No evidence of disease. 